\title{
A Novel Customised Anti Phishing Framework for Mobile Environment
}

\author{
Shaik. Shahid ${ }^{1}$, Dasari Rajesh ${ }^{2}$ \\ ${ }^{1,2}$ Computer Science and Engineering, Rise Group of Institutions, Ongole, India
}

\begin{abstract}
Now a day's many phishy websites are created by the attackers to extract all the personal information from the users. Many people use mobile internet through mobile phones. The proposed anti phishing tool to detect the phishing websites is very helpful to save the users from many fraud websites. The WIFI internet connection is used to deliver the internet service to the mobile device. We extract some main criteria and relates with the protected websites. Thus the similarities between the protected websites and the fraud websites are tested visually by various algorithms. Finally the phishing report is shown how far the obtain website is genuine. The alarm is fixed and produces an alert sound if the given website is phishy website.
\end{abstract}

Keywords: Phishing, Websites, Visualization.

\section{Introduction}

Phishing is a new trend to find the fraud websites form million website pages used now a day's. As the technology improves our security towards it also should be improve accordingly. A common user can access any kind of websites which they need to. But the concept is no one see to it whether we use a genuine website which we access to transact the money and etc. So these ways of attacks mainly happens while transactions when the user gives their personal details, pin numbers and so on. These phishing websites can be found in many ways. The idea to use data mining concept is very useful than other applications, because it says mining a data or information from huge database. Data mining can provide a way how to find these phishing websites with the help of many applications and algorithms

In [1] the author detects the phishing detection system for the e-banking. The paper states that the phishing websites mostly get the e-banking sites and attack their passwords, credit card number, bank account and personal details of the user. He says it's a "New Internet Crime". Comparing with the forma like virus and hacking the phishing is mostly popular now days. In this they introduce a risk assessment model with the help of the fuzzy rule and classification algorithm. This model had six basic categories such as URL\& Domain Identity, Security \& Encryption, Source Code and so on. In [2] its learning how to detect the phishing emails. This was helpful to find the emails which are sent by the attackers. When a user is sent a mail the first thing he should check the richness of the vocabulary, the structure of the page and so on. Email filtering does all these features. The method followed by the author is PILFER. The paper states the learning of overall approach, features and empirical evaluations. Based on this we can find the differences in IP-based URL's, Age of the linked-to domain names, Number of links, HTML emails, Number of dots, Number of domains, contains JavaScript, spam-filter output and etc. The conclusion is based on these they are possible to detect the phishing emails by finding the high accuracy by using spam and specialized filters. These are helpful in learning how to detect the phishing emails.

In [3] the paper explains about the behavioral response to phishing risk. This study of paper reports that the pilot survey of 232 computer users to reveal the legitimate emails. It provides the deeper understanding of the web environment. The main behavior of the phishing sites can be found out by the long URL's and it respond to the user through a intermediate page as linking the main page to the sub pages. The colors will be not standard colors in which its default. The validity of the phishing website may be a short period so we can find the years of the website started and its usage. Many of the phishing websites use PayPal and eBay for the transactions and shopping's. The table listed below is the features of five emails and corresponding web sites from email and web role play.

\begin{tabular}{|c|c|c|}
\hline Email & Legitimacy & Relevant features of email and sites \\
\hline Cognix & real & Regarding work details Link in email: www.cognix.com URL in status bar: http://www.cognix.com \\
\hline NASA & real & Sender is known person Addressed to user Link in email: "this" URL: \\
antwp.gsfc.nasa.gov/apod/astropix.html
\end{tabular}

In [4] the paper describes about the social phishing. This social phishing is fully based on the social network database. It has the public data where it finds the social networks and can collect all the emails to check and to authenticate the web logs. Thus it says it is very easy and very effectively done. It takes the note of each authentication part and gives the report of it in graphs. As countermeasures to combat phishing improves, its said that the phishers in their attacks 


\section{International Journal of Science and Research (IJSR) \\ ISSN (Online): 2319-7064}

Index Copernicus Value (2013): 6.14 | Impact Factor (2014): 5.611

to appear more convincing. As the social phishing attacks underscore the dangers of the public it takes all the personal information's and need to adequate countermeasures.

In [5] the antiphishing strategy has been combined with visual similarity to find the phishing sites and create a report based on it. The algorithm proposed in this method is to get all the sufficient blocks from the given URL and then finding the similarities between the protected website and the given website address. Finally it proposed using visual similarity assessment and finds the report by testing websites like eBay, PayPal and so on. In many proposed algorithms they fail to find the phishing websites, but they tried it to a mark upto $50 \%$ still they can't succeed. Many proposed algorithms used association rule and fuzzy algorithms. They are the new mining algorithms in this fast moving world. The association rules for mining the large database is very useful for business websites and marketing.

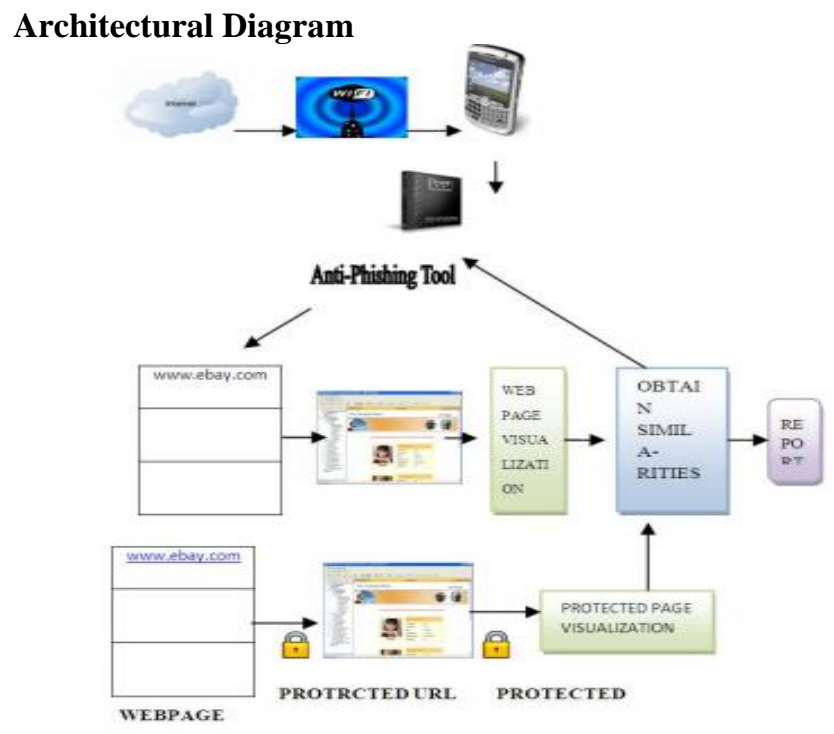

\section{Related Work}

Web users have been suffering from phishing attacks since their first appearance in 2003. Researchers have proposed many solutions (such as alert protection and phishing detection) to defend against phishing attacks.

Alert protection is a simple notification when a user is entering sensitive information. Kirda et al. proposed AntiPhish [9], which tracks the sensitive information of a user and generates warnings whenever the user attempts to give away this information to a website that is considered untrusted. However, this scheme cannot automatically check and detect phishing attacks. Instead, users have to judge by themselves after being warned.

In addition, many phishing detection tools have been designed for phishing on PC web pages. Based on the methodology used, they can be generally categorized into two groups: heuristics schemes and blacklist schemes. Heuristics schemes outperform blacklist schemes since they can deal with new phishing sites without the need of waiting for update. Usually, heuristics schemes for phishing detection utilize other techniques such as machine learning techniques [10], [11], [12] and search engine [12], [13] CANTINA [13] is a content-based approach to detecting phishing websites, and it adopts TF-IDF information retrieval algorithms. Garera et al. [10] proposed a heuristicsbased scheme which identified several generic features of phishing URLs, and used these features in a logistic regression classifier. CANTINA+ [12] is a comprehensive feature-based solution for phishing web page which combines machine learning and search engine techniques. However, existing heuristics used in phishing detection are all based on features extracted from HTML source code. As we showed in section III, HTML source code should not be trusted since it may not reflect the actual content presented to users.

Based on the assumption that the most spoofing phishing sites are those whose visual appearances look identical or very similar to authentic sites [14], [15], several similarity based phishing detection approaches are proposed. SpoofGuard [16] uses URLs, images, links, and domain names to check the similarity between a given page and the pages previously stored. Afroz et al. proposed PhishZoo [17] that uses the profiles of trusted websites' appearances built with fuzzy hashing techniques to detect phishing. PhishZoo makes profiles of sites that consist of fuzzy hashes of several common content elements (e.g. URL, images, most used texts, HTML codes, script files, etc.), which are related to their structure and appearance. They further enhanced their phishing detection scheme by adding displayed images into profiles and utilizing SIFT image-matching algorithm [18] However, similarity based approaches cannot detect phishing sites with different appearances.

GoldPhish [8] utilizes optical character recognition (OCR) technique for phishing detection on PC browsers. OCR is used to extract text from images found on web pages, such as the company logo, and then it is compared to the top ranked domains from Google' s search service. However, OCR performance on PC is demonstrated to be limited in both speed and accuracy. And our lightweight scheme works with mobile browser and does not depend on external search engine.

Mobile Phishing is emerging as a significant threat for mobile users. iPhish [5] discusses the weaknesses caused by the hardware limitation of mobile devices. Felt et al. [4] examined the mobile phishing threats by detailing several phishing attack models during control transfers. In terms of solutions for mobile phishing, we only found one piece of work proposed by Jie et al. [19], in which they load hooks into $\mathrm{iOS}$ so that the system interrupts the user when sensitive information is being entered into applications not in the whitelist, and prompts the user to decide whether to continue or not. However, this idea is quite similar to AntiPhish [9], which only provides a warning, rather than detection and defense.

\section{Proposed Work}

The module description of the phishing tool is classified based on visualization. The modules are based on the data mining algorithms in which the tool can be faster and protective. Each module has different similarities and it can 


\section{International Journal of Science and Research (IJSR) \\ ISSN (Online): 2319-7064}

Index Copernicus Value (2013): 6.14 | Impact Factor (2014): 5.611

be found out when the modules are being match with each other.

\section{Obtain URL}

The URL is the website address which the user enters in the address bar. When the mobile hand set is connected to internet, it extracts the URL from address bar and gives that URL to next modules.

\section{Web page Extractor}

In this module it extracts the web page with the help of given URL. The URL opens the corresponding website and enters into it. It consists of all styles, images, fonts and the corresponding details of that website. This web page is used for the visualization and divides the web page into categories and sub categories.

\section{Web page Visualization}

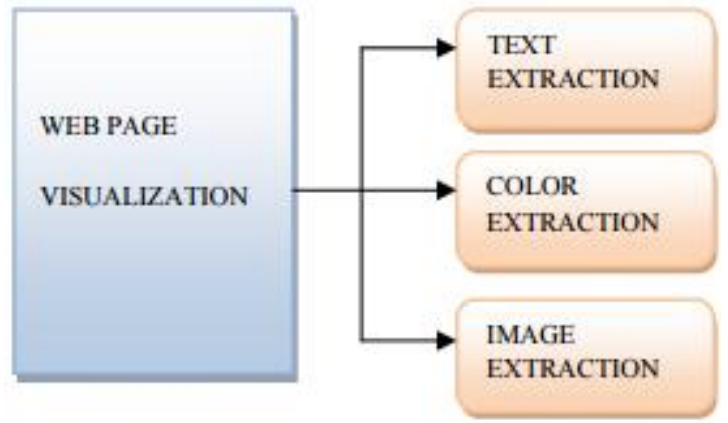

Block Diagram of Webpage Visualization

Visualization technique is the most powerful devices for identifying patterns hidden in data. We extract three main extraction modules which is necessary to find the phishing sites by visualization. The classifications of visualization are

1. Text extraction

2. Color extraction

3. Image extraction

The text extraction will extract all the text contained in the webpage blocks. The text extraction can be further classified into fonts, border text, style and alignment. We get respected values for this classification module. The color extraction will extract all the colors contained in the webpage.

The color extraction can also be further classified into background colour, foreground colour and content color. We get the respected values for this module.

The image extraction will extract the entire image contained in the webpage. The image extraction can be classified as image color, image type and image size. We get the respected valued for this module.

\section{Similarities}

We collect the module values for both the obtained website and the protected website from the above modules. Now compare both the values and find the similarities between the websites.

\section{Report}

With the help of all the similarities we generate a report and if the report is negative we alert a sound alarm and if the report is positive we give the web page to the user.

\section{Conclusion}

The main aim of this paper is to make user safe and a secure access to the mobile internet. These kind of tools had been implemented in early days by WAP servers, but now a day's our technology had been improved. Thus we use wifi, it's now a fast internet connection and can access in all places without any device. So its easy for the mobile internet and the tool and secure user in all ways and will be helpful also. In future ways can test this phishy websites through many ways according to our technology development. The future works can be to fix the anti virus also into the tool in which the user will be comfort to access all pages and be secure.

\section{References}

[1] Intelligent phishing detection system for e-banking using fuzzy data mining by Maher Aburrous, M.A. Hossain, Keshav Dahal, Fadi Thabtah in 2010.

[2] Fette Ian, Sadeh Norman, \& Tomasic Anthony (2006). Learning to detect phishing emails. Institute for Software Research International.

[3] Behavioral response to phishing risk by Julie S. Downs, Mandy Holbrook and Lorrie Faith Cranor.

[4] Liu, W., Deng, X., Huang, G., \& Fu, A. Y. (2006). An anti-phishing strategy based on visual similarity assessment, published by the IEEE computer society 1089-7801/06 IEEE. Internet Computing IEEE.

[5] Phishing Attack Detection by Using a Reputable Search Engine by Robert Ma, Electrical and Computer Engineering Department University of Toronto

[6] http://www.phishtank.com/phish archive.php

[7] Adida, B., Hohenberger, S., \& Rivest, R. (2005). Lightweight encryption for e-mail. In USENIX steps to reducing unwanted traffic on the internet workshop (SRUTI).

[8] Witten, I. H., \& Frank, E. (2005). Data mining: Practical machine learning tools and techniques (3rd ed.). San Francisco, CA: Morgan Kaufmann.

[9] WEKA - University of Waikato, New Zealand, EN (2006). Weka - Data Mining with Open Source Machine Learning Software in Java. Available from http://www.cs.waikato.ac.nz/ml/weka (2006/01/31).

[10]Anti-Phishing Working Group (2007). Phishing Activity Trends Report. Available from http:// antiphishing.org reports/ apwg_report_sep 2007_final. pdf.

[11] Persson Anders (2007). Exploring phishing attacks and countermeasures. Master Thesis in Computer Science, Thesis No: MCS-2007:18. 